\title{
Synthèse régionale des problèmes phytosanitaires de la culture de la tomate à la Martinique (Antilles françaises)
}

\author{
B Hostachy 1, J Mutz 1, P Cadet 2 \\ 1 Service de la protection des végétaux, BP 241, 97257 Fort-de-France cédex, \\ ${ }^{2}$ ORSTOM nématologie, BP 8006, 97259 Fort-de-France cédex, Martinique, France
}

(Reçu le 15 février 1990; accepté le 16 janvier 1991)

\begin{abstract}
Résumé - Une enquête pluridisciplinaire (mycologie, bactériologie, nématologie, virologie et entomologie) sur les contraintes parasitaires de la culture de la tomate a été réalisée à la Martinique. Les résultats ont été étudiés par rapport à la situation géographique de la parcelle sur l'île, au moyen d'une analyse factorielle discriminante après transformation des effectifs en classes d'abondance. Les facteurs 1 et 2 permettent de répartir les champs en 3 sousensembles : Nord Caraïbe, Nord Atlantique et un regroupant Centre et Sud. Ce sont les variables «Mines", "Septoria", "Erwinia", "Criconemella» (facteur 1) et "Acariens" ou "Xiphinema» (facteur 2) qui expliquent la position de ces nuages. Le facteur 3 permet de différencier les zones Centre et Sud, notamment à partir de la variable "Phoma". Le profil parasitaire de chaque région a été établi. Les liaisons significatives entre les variables se répartissent autour d'un trinôme constitué par le nématode Xiphinema, la bactérie Xanthomonas et le symptôme "tache». Des méthodes de lutte phytosanitaire raisonnées au moyen d'itinéraires techniques adaptés, pourraient être développées pour chacune de ces zones, en s'appuyant sur les relations particulières qui apparaissent entre les différents agents pathogènes.
\end{abstract}

Caraïbe / Martinique / tomate / nématode / bactérie / insecte / champignon

Summary - Regional synthesis of tomato diseases in Martinique (French West Indies). A pluridisciplinary survey (mycology, bacteriology, nematology, virology and entomology) of tomato diseases was conducted in Martinique. Results were described in respect to the geographical localization of the field on the island (table I) with a factorial discriminant analysis after data transformation in abundance classes (table II). Factors 1 and 2 permitted classification of the fields into 3 areas: North Carribean, North Atlantic and one for the Center and the South (fig 1). The variables "Mines", "Septoria", "Erwinia", "Criconemella" (Factor 1 and "Acarians" or "Xiphinema" (Factor 2) explain the position of the areas (table III). Factor 3 allows separation of the Center from the South area, mainly according to the "Phoma" variable (table III, fig 2). Pest and parasite complexes in each area have been described (fig 3). The relationships among variables are distributed around a triangular pattern consisting of the nematode Xiphinema, the bacteria Xanthomonas and the "spot" symptom (fig 4). Technical advice for integrated control methods should be developed for each area, using the particular relationships that exist among the different pathogens.

Carribean / Martinique / tomato / nematode / bacterium / insect / fungus

\section{INTRODUCTION}

Depuis que le flétrissement bactérien dû à Pseudomonas solanacearum a pu être maîtrisé à la suite de l'introduction de la variété résistante Caraïbo et grâce à l'extension du réseau d'irrigation, la culture de la tomate s'est considérablement étendue et intensifiée à la Guadeloupe et à la Martinique (Anaïs, 1986). Cette situation a contribué à amplifier et à diversifier les problèmes phytosanitaires, d'autant plus que les nouvelles zones cultivées n'offrent pas toujours des conditions pédo-climatiques bien adaptées à cette culture. Afin de recenser et de mesurer l'évolution des maladies de la tomate, une enquête a été réalisée à la Guadeloupe et à la Martinique par le service de la protection des végétaux, simultanément en mycologie, bactériologie, nématologie, virologie et entomologie. 
Les problèmes dont l'importance dépasse le cadre régional sont généralement résolus au moyen de la sélection variétale (Laterrot, 1989). Mais l'aspect commercial qui prolonge inévitablement la création d'une variété nouvelle écarte la prise en compte de problèmes particuliers qui ne concernent que des zones réduites de culture, et de ce fait, ne représentent pas un débouché suffisant pour les semences. Pour pallier cet inconvénient et faire face aux situations parasitaires les plus variées, les résultats obtenus dans tous les domaines étudiés ont été analysés globalement et par écosystème. Cette démarche devrait nous permettre de jeter les bases de nouvelles stratégies régionales de lutte raisonnée, mieux intégrables dans les divers systèmes de culture de la Martinique et des îles voisines.

\section{MATÉRIEL ET MÉTHODES}

Au cours de l'année 1988, 70 champs de tomate, dont la surface varie de $150 \mathrm{~m}^{2}$ à 1,5 ha (moyenne : 4300 $\mathrm{m}^{2}$ ) ont été visités durant la période la moins humide entre les stades "fruits formés" et la première semaine de récolte. La grande majorité se situent au Nord de la Martinique, zone traditionnelle de culture de la tomate (tableau 1). II s'agit pratiquement toujours du cultivar Caraïbo.

Pour chaque parcelle, l'importance des taches foliaires d'origine bactérienne ou fongique et celle des galeries foliaires creusées par les larves de mouches mineuses a été évaluée sur 25 pieds pris au hasard, en observant les 6 premières feuilles à partir de l'apex, selon une échelle à 3 niveaux : 0 : aucune tache, 1 : taches couvrant moins du quart de la surface foliaire, 2 : taches couvrant plus du quart de la surface foliaire. Ceci revient à attribuer à chaque parcelle une note comprise entre 0 et 300 .

Afin d'identifier les parasites aériens, des échantillons (feuilles, fruits ou tiges) de plantes malades ont été prélevés pour être étudiés au laboratoire. Ces fragments végétaux sont préalablement désinfectés superficiellement à l'aide d'un coton imbibé d'alcool à $95^{\circ}$. Des isolements mycologiques sont réalisés à partir de morceaux de $5 \mathrm{~mm}$ mis à incuber à $27^{\circ} \mathrm{C}$ sur milieu S coulé en boîtes de Petri (Messiaen et Lafon, 1970). Les bactéries sont isolées par dilacération de tissu végétal dans de l'eau distillée stérile. Les suspensions ainsi préparées sont étalées en boîtes de Petri, sur milieu levure, peptone, glucose, agar (LPGA) et sur milieu de Kelman (Kelman, 1954). Les boîtes sont mises à incuber à $27^{\circ} \mathrm{C}$ et caractérisées par des tests biochimiques classiques (réaction de Gram, type respiratoire, métabolisme du glucose, réduction des nitrates, utilisation du tréhalose, du malonate, pigments fluorescents, dégradation des pectates). Les virus sont identifiés par le laboratoire du Groupement régional d'intérêt scientifique phytosanitaire de Montfavet (France). Les chenilles et les pupes sont élevées au laboratoire dans des boîtes grillagées à $25^{\circ} \mathrm{C}$, pour être déterminées au stade papillon (Noctuidae) ou mouche (Liriomyzae).

En ce qui concerne les parasites telluriques, 4 pieds de tomate complets dressés et végétativement actifs, sont arrachés au hasard avec la motte de terre, selon une diagonale de la parcelle. Les nématodes sont extraits du sol et des racines par les méthodes de Seinhorst $(1950,1962)$. L'infestation du champ est déterminée par la moyenne des 4 mesures, rapportée au $\mathrm{dm}^{3} \mathrm{de}$ sol ou au $\mathrm{g}$ de racine sèche. La présence de Pseudomonas solanacearum dans le collet des plantes est détectée au laboratoire de bactériologie de I'INRA de Guadeloupe par un méthode sérologique ( $P$ Prior, communication personnelle). Si la bactérie est présente dans l'un des pieds, la parcelle est considérée comme contaminée.

Compte tenu des disparités entre les échelles de mesure des différentes variables qui représentent les parasites ou les symptômes observés, les effectifs ont été transformés en classe d'abondance (tableau II). Pour chaque variable, les limites des classes ont été définies avant l'analyse statistique en fonction de la gravité potentielle du symptôme ou des dégâts occasionnés par l'agent pathogène.

Le tableau de données comporte 14 colonnes : 13 variables instrumentales (tableau II) plus une variable descriptive : la localisation géographique de la parcelle, et 70 lignes (champs visités). II a été étudié au moyen d'une analyse factorielle discriminante (Cuany et Rodolphe, 1980; Logiciel STAT-ITCF), méthode qui renforce la mise en évidence des différences entre les groupes prédéfinis que sont le Nord-Atlantique, le Nord-Caraïbe, le Centre et le Sud.

Les liaisons entre les variables (agents pathogènes, symptômes ou localités) ont été recherchées à l'aide du test du $\chi^{2}$ effectué sur les tableaux de contingence constitués avec chaque couple de variables.

\section{RÉSULTATS}

Parasites et symptômes fréquemment observés : - symptôme "tache" : nécrose foliaire d'origine indéterminée, manifestation d'une attaque

Tableau I. Répartition des champs de tomate étudiés en fonction de leur position géographique sur l'île de la Martinique.

Localisation

Nombre de champs visités

$\begin{array}{lr}\text { Nord-Caraïbe } & 39 \\ \text { Nord-Atlantique } & 16 \\ \text { Centre } & 5 \\ \text { Sud } & 10\end{array}$


Tableau II. Intitulé des variables et définition des limites des classes d'abondance.

\begin{tabular}{|c|c|c|c|c|c|c|c|}
\hline Variables & $N^{\circ}$ & Mod & Cl d'abondance & Variables & $N^{0}$ & Mod & Cl d'abondance \\
\hline Mines & 12 & $\begin{array}{l}1 \\
3\end{array}$ & $\begin{array}{l}\text { Gravité }<70 \text { * } \\
71<\text { Grav }<200 \\
\text { Gravité }>200\end{array}$ & Tache & 22 & $\begin{array}{l}1 \\
3\end{array}$ & $\begin{array}{l}\text { Gravité < } 20 \text { * } \\
21<\text { Grav < } 100 \\
\text { Gravité > } 100\end{array}$ \\
\hline Septoria & 3 & $\begin{array}{l}1 \\
2\end{array}$ & $\begin{array}{l}\text { Absence } \\
\text { Présence }\end{array}$ & Phoma & 4 & $\begin{array}{l}1 \\
2\end{array}$ & $\begin{array}{l}\text { Absence } \\
\text { Présence }\end{array}$ \\
\hline Pseudomonas & 5 & $\begin{array}{l}1 \\
2\end{array}$ & $\begin{array}{l}\text { Absence } \\
\text { Présence }\end{array}$ & Erwinia & 6 & $\begin{array}{l}1 \\
2\end{array}$ & $\begin{array}{l}\text { Absence } \\
\text { Présence }\end{array}$ \\
\hline Xanthomonas & 7 & $\begin{array}{l}1 \\
2\end{array}$ & $\begin{array}{l}\text { Absence } \\
\text { Présence }\end{array}$ & Acarien & 8 & $\begin{array}{l}1 \\
2\end{array}$ & $\begin{array}{l}\text { Absence } \\
\text { Présence }\end{array}$ \\
\hline $\begin{array}{l}\text { Helicotylenchus } \\
\text { par } \mathrm{dm}^{3} \text { de sol }\end{array}$ & 9 & $\begin{array}{l}1 \\
2 \\
3\end{array}$ & $\begin{array}{l}\text { Absence } \\
1-500 \\
>500\end{array}$ & $\begin{array}{l}\text { Criconemella } \\
\text { par } \mathrm{dm}^{3} \text { de sol }\end{array}$ & 10 & $\begin{array}{l}1 \\
2\end{array}$ & $\begin{array}{l}\text { Absence } \\
\text { Présence }\end{array}$ \\
\hline $\begin{array}{l}\text { Xiphinema } \\
\text { par } \mathrm{dm}^{3} \text { de sol }\end{array}$ & 11 & $\begin{array}{l}1 \\
2\end{array}$ & $\begin{array}{l}\text { Absence } \\
\text { Présence }\end{array}$ & $\begin{array}{l}\text { Meloidogyne } \\
\text { par g de racine }\end{array}$ & 12 & $\begin{array}{l}2 \\
3\end{array}$ & $\begin{array}{l}1<500 \\
500-10000 \\
>10000\end{array}$ \\
\hline $\begin{array}{l}\text { Rotylenchulus } \\
\text { par g de racine }\end{array}$ & 13 & $\begin{array}{l}1 \\
2\end{array}$ & $\begin{array}{l}<2000 \\
>2000\end{array}$ & Localisation & 142 & $\begin{array}{l}1 \\
3 \\
4\end{array}$ & $\begin{array}{l}\text { Nord Caraïbe } \\
\text { Nord atlantique } \\
\text { Centre } \\
\text { Sud }\end{array}$ \\
\hline
\end{tabular}

* Cf Matériel et méthodes

d'agent pathogène aérien, qui permet de caractériser l'aspect général de la parcelle;

- entomologie : mouche mineuse : Liriomyza sativae; acariens : Aculops lycopersici et différentes espèces de Tétranyques;

- mycologie : Septoria lycopersici et Phoma destructiva;

- bactériologie : Pseudomonas solanacearum, Erwinia chrysanthemi et $E$ carotovora; Xanthomonas campestris pv vesicatoria;

-nématologie : Helicotylenchus ( $H$ erythrinae, $H$ retusus et $H$ dihystera) Criconemella ( $C$ onoensis, $C$ ornata), Xiphinema ( $X$ vulgare, $X$ spp), Meloidogyne ( $M$ arenaria, $M$ spp) et Rotylenchulus reniformis.

Le tableau analysé n'intègre pas la totalité des agents pathogènes identifiés dans les champs de tomate de Martinique (Bertrand, et al, 1990). Ceux qui apparaissent très rarement et en très faible quantité n'ont pas été pris en considération, comme par exemple les nématodes Hoplolaimus seinhorsti, Pratylenchus brachyurus et $\mathrm{Pa}$ ratrichodorus sp ou encore les virus PVY et TMV.

\section{Caractéristiques parasitaires des 4 zones de culture étudiées}

Le plan factoriel constitué avec les facteurs 1 et 2 de l'analyse discriminante décrit environ $80 \%$ de la variabilité totale. Dans ce plan, les points correspondant aux champs étudiés se répartissent en 3 ensembles (fig 1) :

- le premier ensemble regroupe la plupart des parcelles du Nord-Atlantique dont les abscisses sont négatives et les ordonnées le plus souvent positives;

- le second ensemble regroupe les parcelles du Nord-Caraïbe, dont les ordonnées sont généralement négatives;

- le troisième ensemble regroupe les parcelles du Centre et du Sud dont les abscisses et les ordonnées sont positives à une exception près.

Les zones de culture du Centre et du Sud se différencient selon le facteur 3 qui n'exprime que $18 \%$ de la variabilité totale (fig 2). Les parcelles du Centre ont des coordonnées factorielles positives, alors que celles du Sud sont négatives ou 


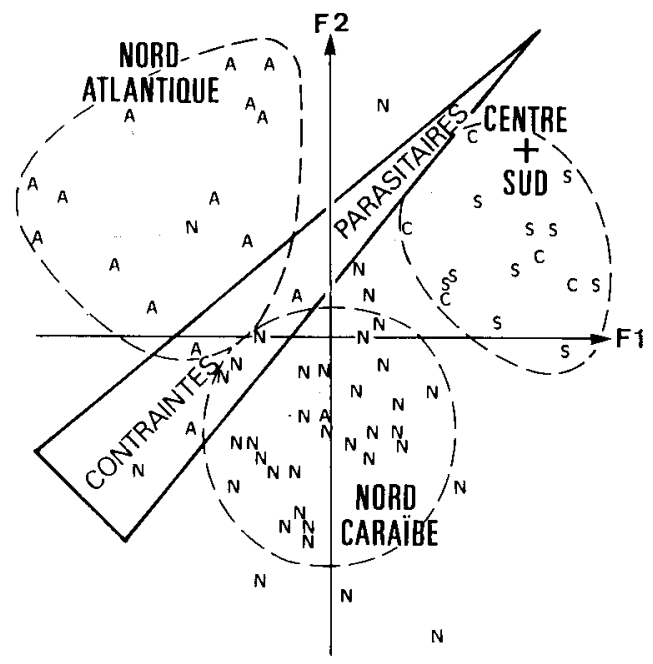

Fig 1. Les points correspondant aux différentes parcelles de tomates sont projetés sur le plan factoriel $F_{1} \times F_{2}$, où ils constituent 3 ensembles bien identifiés: Nord-Atlantique (A), Nord-Caraïbe (N) et Centre (C) + Sud (S) .

voisines de 0 . (Pour les 2 autres zones de culture du Nord, les coordonnées se répartissent uniformément entre les valeurs négatives et positives du facteur 3 ).

La situation des ensembles précédemment décrits dans le plan factoriel $F_{1} \times F_{2}$ provient de la variabilité plus ou moins importante de certaines variables. Plus la corrélation entre la variable et le facteur est élevée, plus cette variable participe à la séparation des ensembles le long de l'axe représentant le facteur (tableau III);

- l'axe 1 est corrélé négativement avec les variables "Mines», «Septonia», "Erwinia» et "Criconemella", moins nettement avec "Tache" et positivement avec «Pseudomonas»;
- l'axe 2 est négativement corrélé avec "Xanthomonas» et «Helicotylenchus», mais surtout avec "Acarien» et "Xiphinema»;

- l'axe 3 est corrélé négativement à la variable "Phoma", plus ou moins aux variables "Helicotylenchus" et "Tache». II est positivement corrélé à la variable "Rotylenchulus».

L'analyse simultanée de la position des ensembles de points dans le plan factoriel et des corrélations entre les variables et les axes de ce plan, permet de dresser le "profil parasitaire» de chaque région ou groupe de régions. Les profils recensés ont été illustrés à l'aide d'histogrammes représentant les fréquences d'apparition (en pourcents) de chacune des classes d'abondances des variables significatives (tableau III et fig 3 );

- les contraintes parasitaires sont moins nombreuses au sud de l'île (regroupement Centre et Sud) qu'au nord (Nord-Caraïbe plus NordAtlantique). Les différences portent essentiellement sur l'abondance de Meloidogyne, de Xanthomonas, de Septoria et sur le fait qu'il y a un peu moins de Pseudomonas;

- la côte caraïbe se distingue de la côte atlantique (regroupement des zones Nord-Atlantique, Centre et Sud) par la présence de Xiphinema, d'acariens, de Xanthomonas et d'Helicotylenchus;

- le nord et le sud de la Côte atlantique se différencient par la présence d'Erwinia, de mines, de Meloidogyne et de Criconemella ou de Septoria;

- Rotylenchulus est plus abondant au centre qu'au sud alors que Phoma et Helicotylenchus y sont, à l'inverse, moins abondants.

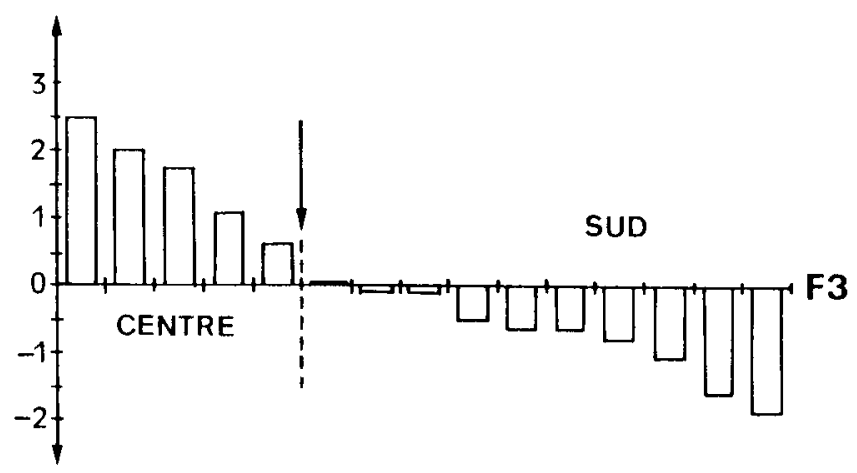

Fig 2. Représentation fonctionnelle du troisième facteur pour les parcelles Centre et Sud disposées selon l'abscisse par valeur factorielle décroissante.

Fig 3. Distribution des abondances des principales caractéristiques parasitaires ou symptômes distinctifs des différentes régions ou groupes de régions de la Martinique, figurés sur la carte et l'histogramme par des hachures correspondantes (A: absence, P: présence; XANTHO: Xanthomonas; HELICO SOL: Helicotylenchus par $\mathrm{dm}^{3} \mathrm{de}$ sol; XIPHI SOL: Xiphinema par $\mathrm{dm}^{3}$ de sol; SEPTO: Septoria; ERWIN: Erwinia; MELO R: Meloidogyne par g de racine; CRICO: Criconemella par $\mathrm{dm}^{3}$ de sol; PSEUDO: Pseudomonas; ROTY R: Rotylenchulus par $\mathrm{g}$ de racine. Pour les classes d'abondance, se reporter au tableau II). 


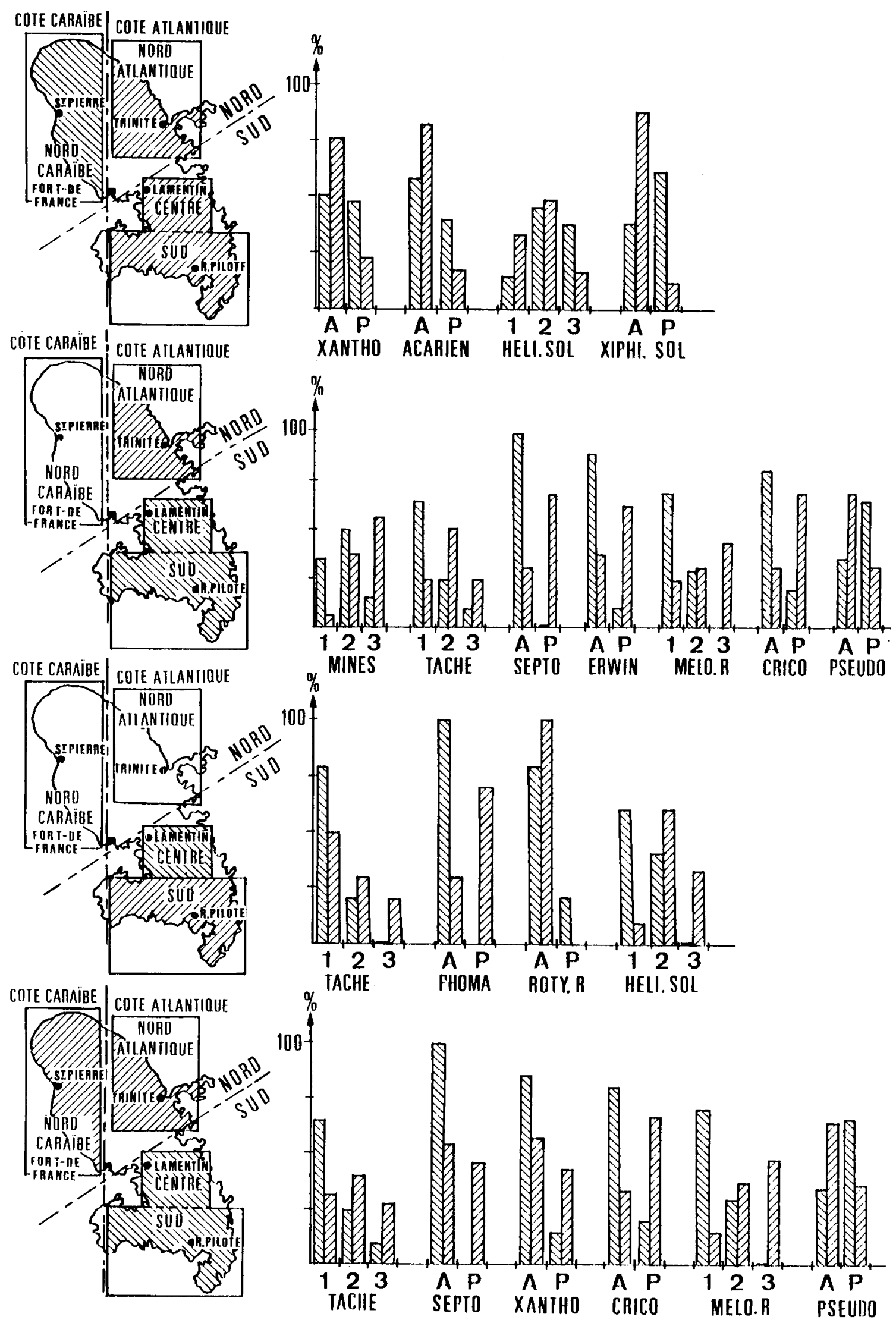


Tableau III. Corrélations entre les variables et les facteurs (les plus significatives sont en italique); $\mathrm{R}$ : racines.

\begin{tabular}{lrrr}
\hline Variables & Facteur 1 & Facteur 2 & Facteur 3 \\
\cline { 3 - 4 } Mines & $-0,9581$ & 0,2759 & $-0,0779$ \\
Tache & $-0,7583$ & $-0,3092$ & $-0,5735$ \\
Septoria & $-0,9309$ & 0,3566 & $-0,0800$ \\
Phoma & $-0,0061$ & $-0,0510$ & $-0,9986$ \\
Pseudomonas & 0,7871 & 0,1176 & 0,6053 \\
Erwinia & $-0,8121$ & 0,4923 & $-0,3139$ \\
Xanthomonas & $-0,4843$ & $-0,7190$ & $-0,4978$ \\
Acariens & 0,0938 & $-0,9941$ & 0,0549 \\
Helicotylenchus & 0,0583 & $-0,7184$ & $-0,6926$ \\
Criconemella & $-0,8640$ & $-0,3734$ & 0,3381 \\
Xiphinema & $-0,3520$ & $-0,9332$ & $-0,0713$ \\
Meloidogyne R & $-0,7266$ & $-0,6446$ & 0,2382 \\
Rotylenchulus R & $-0,7052$ & $-0,2910$ & 0,6468 \\
\hline
\end{tabular}

\section{Les relations entre agents pathogènes, symptômes et régions}

Un certain nombre de liaisons entre variables se sont révélées significatives. Dans tous les cas, l'abondance d'un parasite est positivement corré- lée à celle d'un autre. Les relations entre les variables se répartissent autour d'un triangle constitué par le nématode Xiphinema, la bactérie Xanthomonas et le symptôme "tache" (fig 4). La présence de Xiphinema est elle-même corrélée à celle de Meloidogyne et de Criconemella, entre eux et, en ce qui concerne Meloidogyne, à Rotylenchulus. Ce groupe de nématodes est relié à la zone Nord Caraïbe par son pivot : Xiphinema et par Criconemella; d'autre part à la zone Nord Atlantique par les genres Meloidogyne et Criconemella.

Les 2 autres pivots «tache" et "Xanthomonas" ne sont pas liés à une zone géographique particulière, mais les variables qui s'y rattachent le sont généralement. Le symptôme "tache" est fortement corrélé à "Phoma", lui même corrélé à la localisation sud. Si "Mines" est faiblement associé à "Phoma" dans la zone sud, il est aussi faiblement associé, dans la zone Nord Atlantique, au pivot "Xanthomonas". Ce dernier est lié à un ensemble constitué par "Septoria", "Erwinia", "Acarien" (plus particulièrement dans le Nord Atlantique), mais aussi à la composante «nématode».

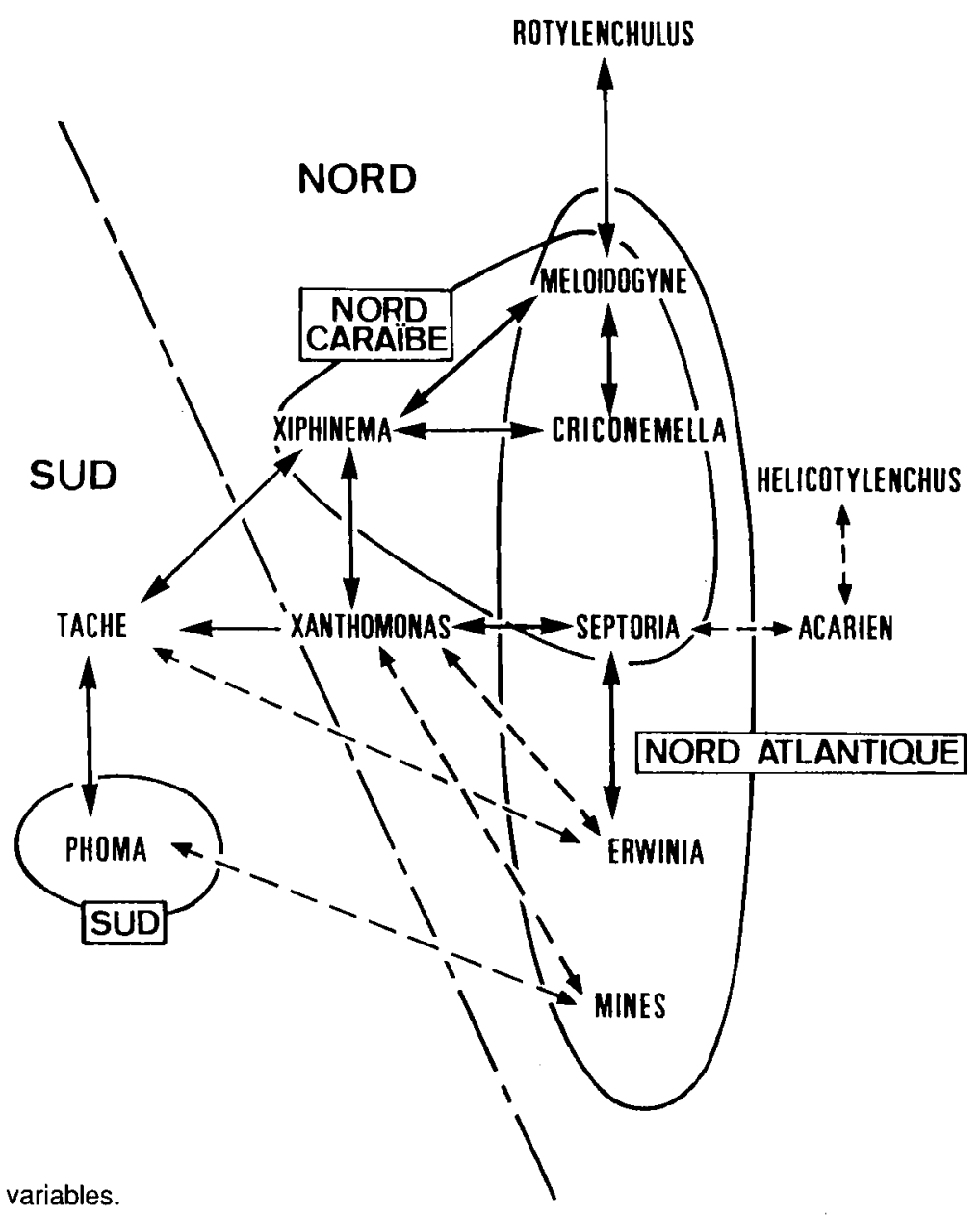

Fig 4. Relations entre les variables. 


\section{DISCUSSION}

\section{Répartition des problèmes phytosanitaires}

Les 4 zones de culture de la tomate définies à partir de la position géographique de la parcelle étudiée sur l'île, peuvent être réunies en 2 grands ensembles selon leur "profil parasitaire»: le Nord qui regroupe le Nord-Atlantique et le Nord-Caraïbe, le Sud, qui regroupe les zones Centre et Sud. Les contraintes parasitaires sont beaucoup plus nombreuses dans le premier ensemble, zone traditionnelle de culture de la tomate, que dans le second. La faune nématologique, les flores mycologique et bactérienne y sont plus variées et provoquent un grand nombre de maladies (fig 3).

Cependant, si la nature des contraintes est la même pour les 2 composantes de l'ensemble "Nord", la composition du complexe parasitaire diffère entre les zones Nord-Atlantique et NordCaraïbe (tableau IV). Au plan nématologique, Meloidogyne est omniprésent au Nord, mais les 2 sous-ensembles se distinguent à partir des autres genres comme Xiphinema légèrement moins fréquent côté atlantique que côté caraïbe. La même situation existe au plan entomologique entre les acariens et les mouches mineuses, ou encore au plan bactériologique entre Erwinia et Xanthomonas, et enfin au plan mycologique pour Septoria plus particulièrement inféodé à la zone Nord-Atlantique.

Tableau IV. Résumé des caractéristiques phytosanitaires de chaque région étudiée (R: racines).

Nord- Nord-

Caraïbe Atlantique Centre Sud

\begin{tabular}{|c|c|c|c|c|}
\hline Mines & & + & & \\
\hline Tache & + & + & & + \\
\hline Septoria & & + & & \\
\hline Phoma & & & & + \\
\hline Pseudomonas & & & + & + \\
\hline Erwinia & & + & & \\
\hline Xanthomonas & + & & & \\
\hline Acariens & + & & & \\
\hline Helicotylenchus & + & & & + \\
\hline Criconemella & & + & & \\
\hline Xiphinema & + & & & \\
\hline Meloidogyne R & + & + & & \\
\hline Rotylenchulus $\mathrm{R}$ & & & + & \\
\hline
\end{tabular}

\section{Étude des relations entre les agents pathogènes:}

Parmi les relations statistiquement identifiées entre les variables, certaines sont de type direct, facilement explicables. Par exemple le symptôme "tache» peut résulter selon la zone climatique de la présence de Phoma ou de Xanthomonas, 2 agents pathogènes qui provoquent effectivement des taches sur le feuillage des tomates (Messiaen, 1975; Blancard, 1988). Bien que Septoria soit capable de produire le même symptôme, la liaison "Septoria-Tache" n'est pas mathématiquement significative.

Par contre, des interactions bien connues et démontrées au laboratoire ne sont pas apparues de manière significative à la suite de l'analyse statistique : c'est le cas de Pseudomonas solanacearum et de Meloidogyne. Les résultats indiquent que la bactérie est plus souvent présente dans les tomates prélevées au sud de l'île, alors que les problèmes de flétrissement ont toujours été plus importants dans la zone traditionnelie de culture au nord de l'île. Ceci provient du fait que notre fréquence n'est pas calculée à partir du nombre de plants flétris dans la parcelle, mais à partir du nombre de fois où cette bactérie a été détectée dans le collet des plantes récoltées pour les analyses bactériologiques et nématologiques, qui sont toujours des plants apparemment sains. Ces résultats confirment que la variété Caraïbo qui est résistante au flétrissement bactérien, héberge $P$ Solanacearum. Cependant, cette résistance diminue lorsque les racines de la tomate Caraïbo sont simultanément attaquées par Meloidogyne; une interaction qui déclenche le flétrissement du plant (Cadet, et al, 1989). Au sud, où Meloidogyne est rare, les pieds de tomate qui sont atteints par $P$ solanacearum ne flétrissent pas et peuvent être prélevés pour les analyses nématologiques (un champ sur 10 a des pieds flétris). Au nord, où Meloidogyne est abondant, les pieds de tomate qui sont également atteints par la bactérie flétrissent et disparaissent avant le stade «fruits formés". (60\% des champs de Caraïbo ont des pieds flétris). Ils ne sont donc plus échantillonnés. Cette situation peut également résulter de la nature des argiles. La montmorillonite présente au Sud inhibe l'action pathogène de $P$ solanacearum (Schmit et al, 1991).

Un certain nombre de relations entre parasites ne sont pas explicables au plan biologique dans l'état actuel de nos connaissances, en termes d'interaction ou de synergie. Elles sont de types «indirect» : 
- liaison nématode-bactérie non tellurique, par exemple entre Xiphinema, nématode du sol, et Xanthomonas, bactérie véhiculée par les graines, mais qui est disséminée plus facilement au Nord-Caraïbe, où les nuits sont chaudes et humides (Timmer, et al, 1987) et où il est plus souvent nécessaire d'irriguer que dans la zone Nord Atlantique,

- liaison bactérie-champignon entre Xanthomonas et Septoria, ou entre Septoria et Erwinia. Dans ce dernier cas, on peut cependant signaler que ces 2 agents pathogènes ont un développement fortement stimulé par l'humidité (Okpala, 1977; Dhanvantari et Dirks, 1987), condition qui existe préférentiellement sur la côte atlantique par rapport à la côte caraïbe plus sèche;

- liaisons nématode-nématode, comme celle qui apparaît entre Criconemella, nématode ectoparasite et Meloidogyne, nématode endoparasite.

Ces liaisons pourraient s'expliquer en admettant que les dégâts occasionnés par un nématode ou un autre parasite tellurique, sensibilisent ou prédisposent la plante hôte aux attaques d'autres agents pathogènes. Cependant, ces relations internématodes ou interorganismes pourraient également provenir de pullulations simultanées mais indépendantes des parasites, car toutes deux induites par le ou les mêmes facteurs abiotiques. À l'appui de cette hypothèse, signalons que la relation MeloidogyneCriconemella a déjà été mise en évidence dans des peuplements de nématodes parasites de la canne à sucre en Afrique de l'Ouest (Cadet et Debouzie, 1990).

- Liaison nématode-sol. C'est un parallèle qui a très souvent été mentionné, mais qui n'est pas expliqué (Hu, et al, 1968; Spaull, 1981; Cadet, 1987). Dans le centre et le sud de l'île où les sols sont généralement très argileux et riches en matière organique, les nématodes sont peu représentés. Alors que dans les sols légers sur ponce au nord, toutes les espèces de nématodes et notamment Meloidogyne, se multiplient abondamment.

Dans un cas, cette relation n'a pas pu apparaître dans notre analyse, car elle ne se manifeste pas au niveau du genre, mais à celui de l'espèce. Ainsi, Helicotylenchus caractérise à la fois le Nord-Caraïbe et le Sud. Mais au nord (andosols sur ponce), il s'agit de : $H$ erytrinae et $H$ dihystera, alors que dans les vertisols au sud, il s'agit exclusivement de $H$ retusus. Cette diversité spécifique au niveau de genre Helicotylenchus n'existe pas en Afrique de l'Ouest (Cadet, 1987; Dick et Spaull, 1982), mais se retrouve dans d'autres îles volcaniques de la Caraïbe (O'Relly et Milian, 1978).

\section{Présence du parasite et importance économique de la maladie}

Le cas de Pseudomonas évoqué précédemment démontre que dans notre étude, ce n'est pas l'importance économique du pathogène qui a été prise en compte, mais son abondance. Dans certains cas, cette importance pourrait effectivement être très secondaire. Criconemella et Helicotylenchus entrent très probablement dans cette catégorie. II convient cependant d'être prudent, car le rôle des parasites n'a pas toujours été analysé de manière approfondie, notamment à travers leur action au sein d'un complexe parasitaire. D'autre part, les facteurs qui déterminent la pathogénie d'un parasite vis-à-vis d'une plante sont susceptibles de changer d'une année sur l'autre, par exemple avec la climatologie. Au cours de l'enquête, certaines pratiques culturales ont pu également conduire à surévaluer ou sousévaluer l'abondance d'un parasite. Au sud, où les traitements antifongiques sont rares, Phoma (peu pathogène) est très abondant, alors qu'au nord, c'est l'inverse.

\section{Avertissement et stratégie de lutte phytosanitaire}

Les relations interorganismes que nous avons identifiées pourraient être utilisées à 2 niveaux :

- d'une part, en vue de simplifier la reconnaissance des maladies qui surviennent au cours du cycle de la plante. À partir du moment où un seul agent pathogène a été détecté, il semble à priori possible de savoir quels seront les autres parasites qui auraient la plus forte chance d'apparaître, compte tenu des liaisons privilégiées qui ont été précédemment identifiées entre ces organismes;

- d'autre part, pour développer des méthodes de lutte raisonnée basées sur la prévention du risque parasitaire, visant à éviter l'apparition des maladies en empêchant le développement des agents pathogènes qui font partie du complexe parasitaire potentiellement capables de se mettre en place dans les conditions écologiques de la zone cultivée. Dans le cas de la Martinique, il apparaît nécessaire de définir au moins 3 itinéraires techniques : un pour le Nord-Ouest, un pour le Nord-Est et un pour le Sud.

\section{Le choix du découpage géographique}

Notre analyse discriminante montre que des points correspondant à des champs de tomate 
situés dans la région Nord-Caraïbe apparaissent dans le nuage de points correspondant à la région Nord-Atlantique et réciproquement. Toutes les parcelles situées dans la même zone géographique ne présentent pas un profil parasitaire rigoureusement identique. Le relief accidenté du nord de l'île génére des microclimats qui influencent localement le développement des parasites; particularité que nous aurions pu isoler en introduisant des variables descriptives supplémentaires topographiques et pédologiques. Autrement dit, le découpage que nous avons utilisé, basé sur la position géographique de la parcelle, ne permet pas de définir un itinéraire technique satisfaisant la totalité des agriculteurs. II est en outre matériellement impossible de traiter individuellement chaque cas. Pour transmettre les informations les plus personnalisées possibles, la solution la plus adaptée pourrait consister à utiliser, comme code d'identification des points sur la carte factorielle, le découpage administratif du territoire utilisé par les techniciens agricoles chargés de conseiller les agriculteurs. Ceci leur permettrait de connaître les champs où il est nécessaire de modifier l'itinéraire technique standard, valable pour la majorité des problèmes parasitaires de leur secteur.

\section{CONCLUSION}

Identifier les contraintes parasitaires d"une culture à échelle d'une île au climat aussi diversifié que celui de la Martinique ne constitue pas un moyen réaliste pour le développement ultérieur de méthodes de lutte capables de satisfaire le plus grand nombre d'agriculteurs. La régionalisation des problèmes offre en revanche une base de travail plus pratique car elle tient compte du fait que les caractéristiques pédoclimatiques jouent souvent un rôle déterminant dans les pullulations de parasites sur une culture. Enfin, grâce à la connaissance des structures des différents complexes parasitaires, le sélectionneur disposera des informations nécessaires pour choisir, parmi les parasites, ceux avec lesquels la plante a le plus de chance d'être confrontée simultanément.

\section{REMERCIEMENTS}

Les auteurs remercient MS Marie-Luce (INRA) et D Duféal (SPV) pour leur collaboration technique, ainsi que $P$ Prior et $A$ Fournet pour leurs conseils lors de la rédaction de cet article.

\section{RÉFÉRENCES}

Anais G (1986) L'approche de solutions à des problèmes phytosanitaires des cultures des Antilles et de la Guyane. Bull Tech Inf 409-411, 449-452

Bertrand P, Hostachy B, Mutz JN (1990) Étude des contraintes parasitaires de la culture de la tomate aux Antilles françaises. Phytoma 414, 55-61

Blancard D (1988) Maladie de la tomate. INRA Paris, PHM Limoges, 212 p

Cadet $P$ (1987) Étude comparative des peuplements naturels de nématodes parasites associés à la canne à sucre. Nematologica 33, 97-105

Cadet P, Debouzie D (1990) Évolution spatiotemporelle d'un peuplement de nématodes parasites de la canne à sucre. Rev Nématol 13, 79-88

Cadet P, Prior P. Steva H (1989) Influence de Meloidogyne arenaria sur la sensibilité de deux cultivars de tomate à Pseudomonas solanacearum (EF Smith) dans les Antilles françaises. Agron Trop 44, 263-268

Cuany A, Rodolphe F (1980) Data of field experiments with nematicides. Rev Nématol 3, 37-50

Dhanvantari BN, Dirks VA (1987) Bacterial stem rot of greenhouse tomato : etiology, spatial distribution and the effect of high humidity. Phytopathology 77 , 1457-1463

Dick J, Spaull VW (1982) Nematode pests of sugarcane. In: Nematology in Southern Africa (Keetch DP, Heyns J, eds) Sci Bull 400, Dep Agric Fish Rep S Afr, 47-57

Hu CH, Tsai TK, Chu HT (1968) The nematode investigation in sugarcane fields of Taiwan and effects of soil fumigation. Proc Int Soc Sugar Cane Technol 13, 1262-1269

Kelman A (1954) The relationship of pathogenicity in Pseudomonas solanacearum to colony appearence on tetrazolium medium. Phytopathology 44 , 693-695

Laterrot H (1989) Intérêt et utilisation des espèces sauvages pour la création variétale. PHM Rev Hortic 295, 13-17

Messiaen CM (1975) Maladies. In : Le potager tropical, cultures spéciales. PUF, 205-222

Messiaen CM, Lafon R (1970) Les maladies des plantes maraîchères. INRA, Paris, $411 \mathrm{p}$

Okpala EU (1977) Effect of concentration, weather and periodicity of application of certain fungicides on Septoria lycopersici (Spreg), leaf blotch disease of tomato at Nsukka, Nigeria. Acta Hortic 53, 64-70

O'Relly JP, Milian JRP (1978) Nematodes parasiting sugarcane in Cuba. Proc Int Soc Sugar Cane Technol 16, 497-507

Schmit J, Prior P, Quiquampoix H, Robert M (1991) Studies on survival and localisation of Pseudomonas solanacearum in clays extracted from vertisols. 7 th ICPPB's proc, Budapest, Hongrie, (sous presse) 
Seinhorst JW (1950) De beteckenis van de toestand van de grond voor het optreden van aan stasting door het stengelaaltje (Ditylenchus dipsaci (Kühn) Filipjev). Tijdschr Plantenziekten 56, 291-349

Seinhorst JW (1962) Modifications of the elutriation method for extracting nematodes from soil. Nematologica $8,117-128$
Spaull VW (1981) Nematodes associated with sugarcane in South Africa. Phytophylactica 13, 175-179

Timmer LW, Marois JJ, Achor D (1987) Growth and survival of Xanthomonas under conditions nonconducive to disease development. Phytopathology $77,1341-1345$ 